\title{
Uveitis-induced Refractory Ocular Hypotony Managed with High-dose Latanoprost
}

\author{
Fariba Ghassemi ${ }^{1}$, MD; Mohammad Reza Niyousha ${ }^{2}$, MD; Narges Hassanpoor ${ }^{3,4}$, MD, MPH \\ Hassan Khojasteh ${ }^{1}$, MD \\ ${ }^{1}$ Retina \& Vitreous Service, Farabi Eye Hospital, Tehran University of Medical Sciences, Tehran, Iran \\ ${ }^{2}$ Retina \& Vitreous Service, Nikookari Eye Hospital, Tabriz University of Medical Sciences, Tabriz, Iran \\ ${ }^{3}$ Ophthalmic Research Center, Shahid Beheshti University of Medical Sciences, Tehran, Iran \\ ${ }^{4}$ Department of Ophthalmology, Tabriz University of Medical Sciences, Tabriz, Iran
}

ORCID:

Fariba Ghassemi: http://orcid.org/0000-0001-9423-9650

Narges Hassanpoor: http://orcid.org/0000-0001-8296-0918

\section{Abstract}

Purpose: To report a case of refractory ocular hypotony due to chronic Behcet's disease with good response to high-dose topical latanoprost.

Case Report: We present a 26-year-old man with a known history of Behcet's disease who developed decreasing vision and severe ocular hypotony that was refractory to multiple treatment modalities including subtenon triamcinolone acetonide, ibopamine, pars plana vitrectomy, and silicone oil injection. We decided to try high-dose topical latanoprost for the management of ocular hypotony based on recent reports. After six months, intraocular pressure (IOP) increased by $5 \mathrm{~mm} \mathrm{Hg}$, became stable at $7 \mathrm{~mm} \mathrm{Hg}$, and remained unchanged at month 24.

Conclusion: High-dose topical latanoprost could lead to significant increase in IOP in uveitis-induced refractory ocular hypotony.

Keywords: Behcet; Hypotony; Inflammation; Latanoprost; Uveitis

J Ophthalmic Vis Res 2020; 15 (3): 408-411

\section{INTRODUCTION}

Ocular hypotony is one of the complications of uveitis and causes substantial visual loss. It occurs

\section{Correspondence to:}

Narges Hassanpoor MD, MPH. Ophthalmic Research Center, Shahid Beheshti University of Medical Sciences, 23 Paidar Fard, Bostan 9, Pasdaran Ave., Tehran, 16666, Iran. Tel: +98-21-55421006 Fax: +98-21-55416134 Email: nargeshassanpoor@gmail.com

Received: 15-03-2019Ａccepted: 14-08-2019

\section{Access this article online}

Website: https://knepublishing.com/index.php/JOVR

DOI: 10.18502/jovr.v15i3.7459 because of hyposecretion of the ciliary body owing to inflammation and increased uveoscleral outflow. In the acute phase of the disease, it is usually reversible; and suppressing the inflammation by corticosteroids or other anti-inflammatory medications may restore the intraocular pressure (IOP). However, in chronic uveitis, long-term inflammation can lead to structural changes such

This is an open access journal, and articles are distributed under the terms of the Creative Commons Attribution-NonCommercial-ShareAlike 4.0 License, which allows others to remix, tweak, and build upon the work non-commercially, as long as appropriate credit is given and the new creations are licensed under the identical terms.

How to cite this article: Ghassemi F, Niyousha MR, Hassanpoor N Khojasteh $\mathrm{H}$. Uveitis-induced Refractory Ocular Hypotony Managed with High-dose Latanoprost. J Ophthalmic Vis Res 2020;15:408-411. 
as growth of tractional membranes on the ciliary body and atrophic changes resulting in long-lasting or irreversible hypotony. Chronic ocular hypotony is associated with sight-threatening complications including hypotony maculopathy, choroidal folds, and optic nerve edema. ${ }^{[1-3]}$ Ocular hypotony is defined as IOP $<8 \mathrm{~mm} \mathrm{Hg}$, and most complications arise when IOP is $<4 \mathrm{~mm} \mathrm{Hg}{ }^{[4]}$

\section{CASE REPORT}

In this report, we present a 26-year-old male with a past medical history of Behcet's disease who developed progressive vision loss and severe hypotonia. He had received $15 \mathrm{mg}$ of methotrexate weekly and $7.5 \mathrm{mg}$ of prednisolone daily as well as multiple injections of subtenon triamcinolone acetonide (TA; $40 \mathrm{mg}$ ). He had also undergone phacoemulsification and posterior chamber intraocular lens placement for cataract in his both eyes. Pars plana vitrectomy with silicone oil injection was performed in his right eye for hypotony. Visual acuity was 20/400 in his right eye and "hand motion" in his left eye. Ocular hypotony persisted despite all these treatments in the absence of active inflammation. Corneal folds and band keratopathy were noted after few weeks. Fundus was poorly visible but it was remarkable for cystic changes in the macular region. B-scan showed a significant serous choroidal detachment due to severe hypotony in both eyes. To increase the IOP, multiple injections of $40 \mathrm{mg}$ of subtenon and $4 \mathrm{mg}$ of intravitreal TA were administered; however, no improvement was observed in vision, IOP status, and serous choroidal detachment. Visual acuity deteriorated because of persistent hypotony maculopathy. Ibopamine (a dopamine agonist) eye drops were used for three months with an increase in IOP of $2 \mathrm{~mm} \mathrm{Hg}$ in both the eyes, but no change in vision was detected.

We discussed the details of our experimental treatment based on published studies with the patient and proceeded with the treatment after obtaining a written consent. Subsequently, highdose latanoprost eye drops (XALATAN, 0.005\%, Pfizer) were administered every 6 hours in both eyes.

One month later, IOP increased to $4 \mathrm{~mm} \mathrm{Hg}$, and at two months, to $7 \mathrm{~mm} \mathrm{Hg}$. After two months of latanoprost treatment, we performed a drug rechallenge test by discontinuing latanoprost for four weeks and then resuming the drug to prove its effect on IOP. After 6 months, IOP was stable at $7 \mathrm{~mm} \mathrm{Hg}$ and remained unchanged even after 24 months. B-scan showed significant improvement in hypotony maculopathy and fluid resolved subretinally (Figures $1 \mathrm{~B}$ and $\mathrm{D}$ ). The patient's vision improved to $20 / 200$ in his right eye and "finger counting" at $1.5 \mathrm{~m}$ in his left eye.

\section{DISCUSSION}

Chronic ciliary body inflammation leads to traction on the ciliary body, atrophic changes of this tissue, and subsequent ocular hypotony. Serous choroidal detachment can occur because of chronic hypotony. If ciliary body traction or detachment is visible on ultrasound biomicroscopy, vitrectomy and membranectomy with or without silicone oil injection may be used to improve the IOP. For our patient, although we performed a vitrectomy with silicone oil injection in the right eye, IOP did not change. In such cases, the rise in IOP may be temporary and mandate reinjection of silicone oil in approximately $50 \%$ of patients. ${ }^{[1-3]}$ Ibopamine is a dopamine agonist that can increase aqueous humor secretion and IOP. Administration of ibopamine eye drops increases the IOP by approximately $2 \mathrm{~mm} \mathrm{Hg}{ }^{[5]}$ In our case, ibopamine improved IOP to a barely measurable $2 \mathrm{~mm}$ $\mathrm{Hg}$. Subtenon and intravitreal injections of TA or systemic corticosteroids may increase IOP in chronic hypotony. ${ }^{[6,7]}$ However, these treatments did not improve results in our patient.

Prostaglandins (PGs) play an important role in aqueous humor dynamics. Latanoprost is a selective $\mathrm{PGF}_{2}$ receptor agonist that can reduce IOP by increasing aqueous outflow mostly by increasing the uveoscleral outflow. It is a potent antiglaucoma treatment when applied once daily. ${ }^{[8]}$ However, when administered topically at high concentrations or intracamerally, PGs $E$ and $F$ can cause miosis and raise the IOP. At the same time, they increase the protein content and white blood cells in the aqueous humor. $^{[9]}$

In chronic uveitis, ciliary body atrophy and consequent aqueous hyposecretion may be difficult to overcome; and decreasing aqueous outflow may be a better way to restore the IOP. High-dose latanoprost may cause some grades of inflammation in trabecular meshwork 

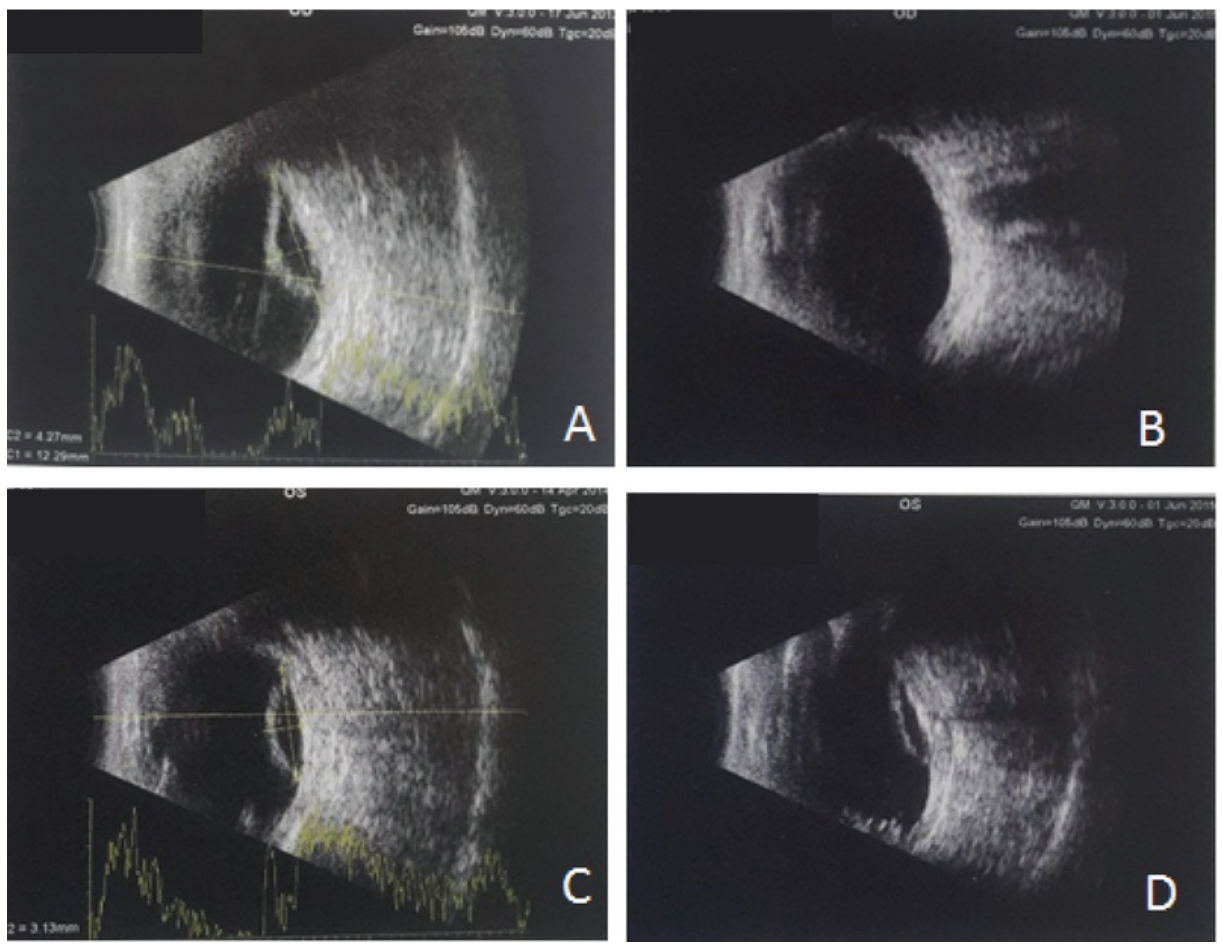

Figure 1. (A) Right eye B-scan before latanoprost treatment. (B) Serous choroidal detachment resolution after treatment with highdose latanoprost in the right eye. (C) Left eye B-scan before latanoprost treatment. (D) Serous choroidal detachment improvement after treatment in the left eye.

and reduce the aqueous outflow. In cases with no tractional membranes on the ciliary body, it may be more practical to increase the uveoscleral outflow. This could be a mechanism by which high-dose latanoprost can raise the IOP in similar cases as our patient. Another less possible mechanism could be improvement of the serous choroidal detachment due to an increase in uveoscleral outflow.

In a report of three cases with uveitic glaucoma, latanoprost had a paradoxical impact on $10 P^{[10]}$ Latanoprost significantly increased IOP, and a latanoprost rechallenge test-performed by discontinuing and then continuing the drugproved that latanoprost could increase the IOP. All patients had uveitis; thus, it can be postulated that in the presence of a severely damaged blood-ocular barrier, latanoprost can have a paradoxical impact on IOP. In that report, latanoprost was administered at a therapeutic once daily dose, and it may be assumed that higher doses, as in our case, may have a more prominent impact on IOP. Higher doses of latanoprost may result in more PG release from the impaired blood-ocular barrier and trabecular inflammation, which can overcome the increase in uveoscleral aqueous outflow and result in raised IOP ${ }^{[10]}$

Latanoprost has reportedly been used in uveitic patients without the risk of central macular edema or recurrence of anterior uveitis. ${ }^{[1]}$ In patients with anterior and intermediate uveitis, low-dose (once daily) latanoprost did not show higher rates of inflammation recurrence when compared to a fixed combination of dorzolamide and timolol. ${ }^{[12]}$ However, similar findings have not been seen in patients with severe posterior uveitis due to Behcet's disease. Therefore, despite it appearing paradoxical to use latanoprost as an IOP-recovering agent in a uveitic patient, its effect might be dependent on the type, severity, and chronicity of uveitis.

It can be concluded that, in some cases, high-dose latanoprost can be administered as an adjuvant treatment for refractory hypotony due to chronic inflammation. Prospective clinical trials to further investigate this can be beneficial. 


\section{Conflicts of Interest}

There are no conflicts of interest

\section{REFERENCES}

1. de Smet MD, Gunning F, Feenstra R. The surgical management of chronic hypotony due to uveitis. Eye 2005;19:60-64.

2. Tran VT, Mermoud A, Herbort CP. Appraisal and management of ocular hypotony and glaucoma associate with uveitis. Int Ophthalmol Clin 2000;40:175-203.

3. Kapur R, Birnbaum AD, Goldstein DA, Tessler HH, Shapiro MJ, Ulanski LJ, et al. Treating uveitis-associated hypotony with pars plana vitrectomy and silicone oil injection. Retina 2010;30:140-145.

4. Pederson JE. Hypotony. In: Tasman W, Jaeger, EA, editors. Duane's clinical ophthalmology. Philadelphia, PA: Lippincott-Raven; 1999; 1-8.

5. Ugahary Le, Ganteris E, Veckeneer M, Cohen AC, Jansen $\mathrm{J}$, Mulder PG, et al. Topical ibopamine in the treatment of chronic ocular hypotony attributable to vitreoretinal surgery, uveitis, or penetrating trauma. Am J Ophthalmol 2006;141:571-573.
6. Sen HN, Drye LT, Goldstein DA, Larson TA, Merrill PT, Pavan PR, et al. Hypotony in patients with uveitis: the Multicenter Uveitis Steroid Treatment (MUST) trial. Ocul Immunol Inflamm 2012;20:104-112.

7. Pederson JE, Mac Lellan HM. Medical therapy for experimental hypotony. Arch Ophthalmol 1982;100:815817.

8. Lim KS, Nau CB, O’Byrne MM, Hodge DO, Toris CB, McLaren JW, et al. Mechanism of action of bimatoprost, latanoprost, and travoprost in healthy subjects. A crossover study. Ophthalmology 2008;115:790-795e794.

9. Abdel-Latif AA. Release and effects of prostaglandins in ocular tissues. Prostaglandins Leukot Essent Fatty Acids 1991;44:71-82.

10. Sacca S, Pascotto A, Siniscalchi C. Ocular complications of latanoprost in uveitic glaucoma: three case reports. J Ocul Pharmacol Ther 2001;17:107-113.

11. Chang JH, McCluskey P, Missotten T, Ferrante P, Jalaludin $B$, Lightman S. Use of ocular hypotensive prostaglandin analogues in patients with uveitis: does their use increase anterior uveitis and cystoid macular oedema? $\mathrm{Br} \mathrm{J}$ Ophthalmol 2008;92:916-921.

12. Markomichelakis NN, Kostakou A, Halkiadakis I, Chalkidou S, Papakonstantinou D, Georgopoulos G. Efficacy and safety of latanoprost in eyes with uveitic glaucoma. Graefes Arch Clin Exp Ophthalmol 2009;247:775-780. 